\title{
How can people save the planet?
}

\author{
How people respond to sustainability challenges is crucial. New findings suggest that when individuals adopt one \\ pro-environmental behaviour, this might affect whether or not they engage in other, related behaviours.
}

\author{
Wokje Abrahamse
}

S cientists are more certain than ever that environmental change is happening and that people's actions are contributing to it. Profound lifestyle changes are needed to avoid the severe impacts of current environmental problems, including climate change. People's individual actions can make a significant contribution ${ }^{1}$. Encouraging people to adopt a range of sustainable behaviours can form an integral part of the solution to environmental challenges ${ }^{2}$. One way is for people to adopt several proenvironmental behaviours one after the other, through a process called behavioural spillover ${ }^{3}$. Writing in this issue, Maki and co-authors examine the potential for behavioural spillover to occur and they analyse under which conditions spillover is more or less likely to happen.

There has been a recent surge in research on what has become known as behavioural spillover. Positive behavioural spillover refers to the notion that when people change one behaviour (for example, they start recycling), this can result in changes in other, related behaviours (for example, they also start reducing food waste). Several theories can explain this phenomenon. For example, when people engage in proenvironmental actions, they may start to see themselves as an environmentally conscious person, which then informs other behaviours. A related explanation is that people strive for consistency in their actions. In contrast, negative behavioural spillover can occur when people refrain from further pro-environmental behaviours because they are already 'doing their bit'. This can be attributed to a process called moral licensing: when we do something good, we worry less about the consequences of doing something harmful.

To date, much behavioural spillover research is correlational, meaning that it looks at bivariate associations between factors, rather than at causality. This approach makes it often difficult to establish cause and effect (that is, did the first behaviour cause people to do a second behaviour, or is it the other way around?). In turn, researchers who use experimental designs can claim causation more confidently - a small, but growing number of authors in spillover research. Such designs allow researchers to compare a group of people who received an intervention (and changed their behaviour) with a control group of people who did not receive it.

The important contribution by Maki and colleagues is that they use meta-analysis to quantify the magnitude of behavioural spillover. They examine several aspects: whether or not engaging in one proenvironmental behaviour results in stronger support for environmental policies, in stronger intentions to engage in a second pro-environmental behaviour, and in the adoption of a second pro-environmental behaviour. The authors find that when people adopt a first pro-environmental behaviour, their intentions to adopt a second pro-environmental behaviour increases. However, the authors also find some evidence for negative behavioural spillover: when people engage in a first pro-environmental behaviour, they then seem less likely to adopt a second pro-environmental behaviour. Here the distinction between intention and actual behaviour is important.

They also analyse whether behavioural spillover depends on the type of intervention to change behaviour. Interventions that strengthen people's intrinsic motivation (doing a behaviour because it is inherently important) are more likely to result in positive behavioural spillover. Interventions focused on extrinsic motivation (doing a behaviour because of something external, such as a reward) tend to produce negative behavioural spillover. In other words, encouraging people to make a commitment to protect the environment ${ }^{4}$ is more likely to produce positive behavioural spillover than using a financial reward. This is a fascinating finding as it suggests that the use of incentives - a commonly used policy tool - may in fact backfire.

Future research on behavioural spillover should build on this meta-analysis by examining behavioural spillover in longitudinal field studies. Longitudinal research on spillover is currently relatively rare. But if the aim is to achieve durable behaviour change, monitoring the dynamics of behavioural spillover over time is important. It would be interesting to examine whether some of the findings of this meta-analysis are also evident or could be replicated in longitudinal research.

We need urgent action to address current environmental problems. The findings of this meta-analysis will be of value to a broad research community, and these findings could and should be used to inform policymaking. This meta-analysis by Maki et al. advances our understanding of how to more-effectively encourage a range of proenvironmental behaviours. It simultaneously addresses an issue of scholarly and societal importance that promises to benefit people and society in a shift towards a more environmentally sustainable future.

Wokje Abrahamse

School of Geography, Environment, and Earth

Sciences, Victoria University of Wellington,

Wellington, New Zealand.

e-mail:wokje.abrahamse@vuw.ac.nz

Published online: 9 April 2019

https://doi.org/10.1038/s41893-019-0273-7

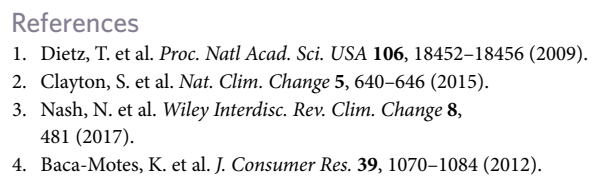

